\title{
Is visuomotor training an effective intervention for children with autism spectrum disorders?
}

This article was published in the following Dove Press journal:

Neuropsychiatric Disease and Treatment

\author{
Manizheh Arabi \\ Alireza Saberi Kakhki ${ }^{1}$ \\ Mehdi Sohrabi' \\ Sakineh Soltani Kouhbanani ${ }^{2}$ \\ Mehdi Jabbari Nooghabi ${ }^{3}$ \\ 'Department of Motor Behavior, Faculty \\ of Sport Sciences, Ferdowsi University of \\ Mashhad, Mashhad, Iran; ${ }^{2}$ Department of \\ Educational Sciences, Educational \\ Sciences and Psychology Faculty, \\ Ferdowsi University of Mashhad, \\ Mashhad, Iran; ${ }^{3}$ Department of Statistics, \\ Faculty of Mathematical Sciences, \\ Ferdowsi University of Mashhad, \\ Mashhad, Iran
}

\begin{abstract}
Purpose: Investigation of autism spectrum disorder (ASD) is somewhat dependent on addressing main core features of ASD. But it is not clear which kind of investigation can effect on more difficulties features. So, this study examines the effect of the visuomotor, motor, and computer-based training programs on social behavior, motor skills, and repetitive behaviors of children with ASD.
\end{abstract}

Patients and methods: Sixty children with ASD aged 6-12 years were recruited and assigned to one of the three experimental groups and the control group (each group $n=15$ ). Training was provided in 30 sessions, scheduled 3 times a week. Social behavior and repetitive behaviors were determined objectively using the observation method, and motor skills were evaluated by the Test of Gross Motor Development-2.

Results: Our results suggested that children in the visuomotor group showed a significant reduction in the repetitive behaviors and an increase in gross motor skill scores in the posttest and follow-up. Also, the results exhibited that motor training group significantly improved in social behavior either in the post-test or follow-up. Although the post-test illustrated a considerable improvement of gross motor skills, this difference was not significant in follow-up. Similarly, no significant change was observed in visual training and control groups in relation to study variables.

Conclusion: Given the improvement of repetitive behaviors and gross motor skills in posttest and follow-up, it seems that this investigation had a positive effect with a good retention effect on two core features of children with ASD. But according to group-based training protocol in motor training group and improvement in social communication, and mutual effect on gross motor skills, it seems that group-based practice can also be used to achieve the benefits of social communication in the investigations.

Keywords: autism disorder, social behavior, repetitive behaviors, gross motor skills

\section{Introduction}

Autism spectrum disorder (ASD) is a neurodevelopmental disorder that is associated with a variety of behavioral deficits. The children with this disease have problem with social behavior, repetitive behaviors, and motor skills. ${ }^{1}$ There is a growing body of research regarding the effect of some training protocols on symptoms of children with ASD such as Feng, Tsai, and Cartledge (2008) showed that social skill training has a positive effect on social communication of a sixth-grade child with autism. ${ }^{2}$ Also Levinson and Reid (1993) revealed that intense exercise training causes decline in repetitive behaviors of children with autism, ${ }^{3}$ and Todd (2012) showed that teaching motor skill to children with autism could enhance motor development of them. ${ }^{4}$ According to these studies, recent research
Correspondence: Alireza Saberi Kakhki Faculty of Sport Sciences, Ferdowsi University of Mashhad, Mashhad, Iran Tel +9805138805418

Fax +9805I 3880738 I

Email askakhki@um.ac.ir 
has shown that the effective treatment of ASD is somewhat dependent on addressing all main critical problem areas of $\mathrm{ASD}^{5}$ which have not been observed in any studies yet. As far as the authors' are concerned, sensory, motor, or sensory-motor difficulties may have effect on core features of children with ASD. A review of the literature suggests that abnormalities of visual perception serve as a possible primary or at least contributory cause that are characteristic of most ASD's behavioral deficits. ${ }^{6}$ In this regard, we compare visual, motor, or visuomotor training protocol and theory on each of the symptoms of children with ASD separately.

Social difficulties are a core feature of identifying autism, which is described by deficits in social cognition, behavior, and communication. ${ }^{7}$ According to the literature, attempts to boost social behavior of individuals with ASDs have focused on either direct social training or peer behavior intervention during playing, which promotes social behavior learning. It has been demonstrated that children with ASD can acquire social skills. ${ }^{8-10}$ All of these interventions are based on the Theory of Mind (TOM) according to which social cognition is a mentalizing activity wherein people should infer other people's intentions. ${ }^{11}$ Nevertheless, the results showed that this positive outcome is task dependent and it cannot be generalized to other social situations in real life of children with ASD. ${ }^{2}$ TOM is a cognitive theory approach which far from causes of sensory or motor difficulties on social problems of ASD. In this regard, Hellendoorn proposed another component that may contribute to the establishment of social behavior in children with ASD, who posited that social behavior is related to the dorsal stream visual processing. ${ }^{11}$ Perception of the face and staring at the eyes, as functions of dorsal visual processing, constitute the main aspects of social communication and understanding of people's intentions or coordination of actions. ${ }^{12}$ Also, part of process feedback produced by eyes and faces is sent to social networks in the brain, which is essential for the development of social behaviors. $^{13}$ In this context, atypical visual processing may impact facial perception and body language, which are vital for social cognition and communication. ${ }^{14,15}$ Also, Gliga et al (2015) demonstrate that early superior visual perception in 9-month-old infants predicts a higher level of autism symptoms at 15 months and 2 years. And, Cheung et al (2018) found that atypical visual perception and core ASD symptoms of social interaction and communication are closely associated during development, and suggest casual links between perceptual and social features.
The notion that atypical visual processing may be related to symptoms of communication difficulties is consist with the embodiment of cognition approach as a perception-action process that needs to pick up relevant social stimuli and adjust an action relative to a situation. ${ }^{11}$ Hence, potential circumstances that may meet visual processing should be considered to increase the possibility of social behavior in children with ASD. On the other hand, it is also claimed that cortical motor system can play a role not only as a controller of basic physical aspects of movement but also in a complex domain as social cognition. ${ }^{16}$ Therefore, motor development may be a marker of and, to some extent, contribute to social interaction difficulties in ASD. Also, it is widely believed that group play/exercise can affect social interaction of typical children or adolescents. ${ }^{17-19}$ But to our knowledge and according to the potential of peer interaction in group-based motor training which can affect TOM there are little investigations to address the effect of small group-based motor training on social performance of children with ASD. For example, Sowa et al (2012) demonstrated that group-based training had a positive effect on social communication of ASD children while individualbased exercise training had positive effect on motor ability. In this regards, Miltenbeeger and Charlap (2013) showed that participate in the athletic skill training and then rules training of children with ASD resulted in increased athletic group play and concomitant increase in speech and this results maintained at $8-16$ weeks follow up. However, generalization to participation in school recess activities did not occur. ${ }^{20}$ Miltenberg did not assess social communication in that study moreover it was a qualitative study which interviewed with children. So, the first aim of this study is to determine which of visuomotor training or group-based motor training can affect social communication of children with ASD according to dorsal stream visual processing difficulties theory and TOM, respectively.

Another core feature of children with ASD is motor development delay, which is commonly reported in children with ASD. ${ }^{21,22}$ We found at least four reviews about ASD and motor training. All of these studies exhibited that motor training had a bearing on aggression, social skills, and stereotypic behaviors, including mixed findings about motor development. ${ }^{5,23-25}$ The limitation of these studies are the single-subject study design as well as conducting aerobic exercise training which could discuss as a functional effect on the nervous system functions or structures. But to our knowledge, there are little investigations which compare the effect of small group motor skill exercise on 
gross motor skills of children with ASD. On the other hand, today, motor developmental delay is assumed to be linked to difficulties in dorsal stream visual perception. ${ }^{12}$ The dorsal stream visual processing plays a crucial role in processing information about spatial relations between objects and their motions. Therefore, it is purported to be involved in position coding and visually guided actions. It is this pathway that is likely to be involved in abnormalities associated with co-orientation, gait, balance, and posture, which are frequently observed in children with ASD. $^{26}$ However, there is scant research regarding the effect of visuomotor training on motor development of children with ASD. Hence, the second aim of this study is to determine which of group-based motor training or visuomotor training can effect on gross motor skills of children with ASD.

Indeed, repetitive behavior is probably a co-occurring mechanism for dorsal stream visual processing difficulties. According to the theory of enhanced perceptual functioning (EPF), autistic individuals struggle with superior, and possibly uncomfortable or overwhelming, processing of low-level visual information. They often adopt behavior strategies that regulate the amount of local information conveyed by an image or scene. ${ }^{27}$ Thus, the main purpose of a study is to regulate the sensory inputs of visual systems to reduce restricted and repetitive behaviors. To compensate for their difficulties in processing visual information in environments, children with ASD may rely on proprioception. ${ }^{28}$ They respond to abnormal visual processing through some odd and repetitive behaviors like head tilt, glancing from the corner of eyes, forward and backward body rock, tow walking, and hand flapping in front of the face, ${ }^{29}$ which are smart ways of information processing that prevail in the world. Therapy programs such as Optometric Vision Therapy (OPT) and motor therapy address this issue. Rose and Torgerson ${ }^{26}$ suggested that OPT can improve the repetitive behaviors of children with ASD. Also, aerobic motor training interventions decline in repetitive behaviors of children with ASD. It is assumed that motor training deterioration is due to physiological changes related to the release of acetylcholine or betaendorphins, ${ }^{24,25}$ even though the lasting effect of both intervention methods is limited. ${ }^{30}$ Thus, the third aim of this study is to investigate the effect of even group-based motor or visuomotor training on repetitive behaviors of children with ASD.

In this study, two investigations, group-based motor and visuomotor training were conducted with the goal of examining their effect on social behavior, gross motor skills, and repetitive behaviors of children with ASD. Also, we conducted a computer-based visual training to control the effect of motor movement which was mutual in motor or visuomotor training groups. To this purpose, we first examined whether the frequency of positive and negative social behaviors, the scores of gross motor skills, and the frequency of repetitive behaviors after 30 sessions of training differed between groups. Second, we compared groups to see which one had affected most core features of children with ASD. Finally, we followed up the enduring effect of interventions for about 2 months. The main purpose of this research was to investigate the effect of 30 sessions of visuomotor, group-based motor and computer-based visual training on core features of children with ASD aged 6-12 years.

\section{Materials and methods Subjects}

Sixty children constituted the participants of this study. A sample of eligible families was recruited from autism institutes where the study was conducted. Families in which the autistic child met the following criteria were considered eligible: (1) the child was in the age range of 6-12 at the time of enrollment, (2) had been diagnosed with an autism spectrum disorder by a licensed psychologist based on the result of the Autism Diagnosis InterviewRevised (ADI-R) and Diagnostic and Statistical Manual of Mental Disorders (DSM-5), (3) had a non-verbal cognitive level of $<60$ (this IQ cut score is based on the cooperative ability of children which was based on pilot study where we assessed the feasibleness of performing this intervention for children with ASD), (4) had trouble processing and integrating visual information as measured by the vision section of the sensory profile (the mean score of visual subscale $>2$ ), and (5) parents were willing to attend 3 weekly sessions (a total of 30 sessions) and refrain from initiating any new visual or motor treatment like medications during the study. In the next step, an appointment was arranged by the school principals, during which the goals and protocols of the study were explained to parents and the contact information of families was obtained. If the child met criteria such as parental consent, child assent, and permission to videotape assessment sessions, he/she would obtain moral standards. After obtaining parental consent, the autism diagnosis was confirmed using the ADI-R and the DSM-5 scales. For children who did 
not have a current cognitive assessment (within the past 6 months), the cognitive level was confirmed by the psychologist. The mean age of subjects was 8.51 years ( $\mathrm{SD}=2.1$; age range: 6-12 years). Further, a complete medical checkup was performed by a physician to ensure the absence of any medical hazard for participating in interventions. The visual acuity was measured monocularly and binocularly with a Snellen chart by an optometrist. All participants had normal acuity. In all groups, children with a history of seizure, brain damage, mental retardation, or other neurological diseases were excluded. The study was approved by the Institutional Review Board and Ethics Committee of Bioethics at the Ferdowsi University of Mashhad (Registration code Of IR.MUM. FUM.REC.1397.14). All parents of children provided written informed consent. The entire study was in keeping with the principles of Declaration of Helsinki.

\section{Intervention}

The participants $(n=60)$ were randomly assigned to either the training groups or the control group (each with 15 subjects). The visuomotor $(n=15)$, motor $(n=15)$, and visual training $(n=15)$ groups had a training frequency of three sessions per week (a total of 10 weeks). Each training session consisted of 10-min warm up, 30-min protocol training, and 10-min cool down. According to the "Applied Behavioral Analysis" method, children's behaviors in training sessions were positively reinforced by verbal feedback. At the same time, the control group $(n=15)$ received routine training.

Participants in the visuomotor group performed certain activities progressively in four different, yet consecutive phases including body awareness and bilateral integration (Lessons 1-9); spatial awareness (Lessons 7-16); peripheral vision training (Lessons 16-23), and saccades and pursuit training (Lessons 23-30). This investigation was conducted based on dorsal visual stream deficit theory. Several references were consulted to provide this visual intervention program. ${ }^{31-34}$ In Table $\mathrm{S} 1$, the exercise of visuomotor group during the intervention has been described. This intervention was conducted by four experienced occupational therapists who had learned the training protocol from an optometrist and a psychologist.

The motor intervention program conducted in the other experimental group was based on Sports, Play and Active Recreation for Kids Active Recreation (SPARK AR) curriculum. $^{35}$ The physical activity may develop motor abilities, health attitude, positive social skills (eg, sharing equipment and cooperation). The motor activities were arranged sequentially from simple to complex. The lessons began with an aerobic activity focused on activities like running, jumping, and jogging to increase the heart rate of children. Then, the fitness activity was followed by skill development time in which children play modified games that improve their physical ability and reinforce movements such as throwing a ball at the wall. We asked four physical education teachers, who were familiar with autism disorder, to help us in this process. This intervention comprised 30 sessions of $1 \mathrm{hr}$, which consisted of warm up, motor exercise aimed at gross motor skills (eg, throwing, catching, kicking), and cool down. This training conduct was group-based (2-3 individuals in each group).

The visual group also performed different visual exercises in video games appropriate for children, which were approved by a psychologist during the same period. These exercises mainly contained eye tracking movements, eye and hand coordination, and cognitive exercises. We used three psychology teachers to monitor and guide children during exercises in fitting with their skill levels.

At the same time, the control group received routine care in their institution. The routine care received during the study period was identical in all groups too. The routine care included non-study-related services such as speech and language services, behavioral interventions, educational program, and other therapies.

\section{Tools}

\section{Repetitive behaviors}

Videotaped observations were used to evaluate the extent of repetitive behaviors. The parts of assessment that were coded included "free play" for 30 mins, as it involved the type of behavior that embraced both objects and people. The "free play" session consists of a structured play session conducted by a psychologist who was familiar with participants which includes a standard series of social communication and play presses in Autism Diagnosis Observation Scale (ADOS) module one which is intended for children who do not use spontaneous phrase speech consistently. For the ADOS play composite, there were a maximum of three items that addressed play (ie, functional play with objects, imagination/creativity, and functional/ symbolic imitation). For example, during the ADOS, the examiner creates a pretend birthday party with a doll, playdough cake, candles, a cup, napkin, and utensils. Toys presented could elicit functional play with objects that might include playing with pop-up toy, stacking blocks, 
or using a play phone. The examiner followed the script design to engage the child while maintaining a flexible but standard format to observe repetitive behaviors. This was coded for the extent of object-grouping behavior, hand flapping, head tilt, body rock, toe walking, and light stares because these behaviors are associated with the regulation of atypical visual processing in $\mathrm{ASD} .{ }^{27}$ The higher frequency of these behaviors was assumed to reflect greater atypical visual processing. A lateral glance was coded in the manner described by Mottron et al:

Fixating on a target with the pupils turned toward an extreme corner of the eye socket, where the head is turned in the opposite direction of the target, in front of it, or in the hemi field corresponding to the target. ${ }^{27}$

Functional lateral glances followed by a reorientation of the gaze so that the object is fixed centrally were not coded. Object-grouping behavior was defined as the spontaneous grouping of two or more objects, which included the liningup of objects, placing objects on top of each other, or putting objects close to each other in a way that is distinctly separated from the rest of the setting. The imitations of the experimenter or object-grouping behavior initiated by the experimenter were not coded. In order to evaluate repetitive behaviors, the method of continuous registration was used and each child was observed individually. Also, in order to increase the accuracy of observation, behaviors were defined explicitly, in addition to live observation, video observation was used to reduce the chance of missing the desired behaviors. Inter-rater reliability was ensured by asking two independent coders to rate $40 \%$ of the tapes. The results suggested $87 \%$ agreement for all behaviors.

\section{Social behavior}

The assessment was conducted in a large room during the working hours of the school, especially between 9.30 and $10.00 \mathrm{am}$, which was the break time. The camera was set up in a steady position covering a large part of the classroom. Although the children were accustomed to being videotaped during common activities all day long, it was necessary to consider the possibility that their behavior might not be influenced. During the session, an experimenter conducted observations and took field notes. She was asked to maintain a thoughtful attitude so that the children would not notice that they were observed.

The analysis was based on social behaviors that occurred during children's plays with their peers in this study. The data collection focused on children's social behavior before, immediately after, and 2 months after training. Two measures were considered in social behaviors: a) positive responses and b) negative responses. In this context, a "response" is defined as any social behavior addressed to a peer player, which follows a social behavior by the same child. ${ }^{36}$ The "response" is categorized as "positive" when the child answers a question, obeys an order, responds with a positive emotion (expressed by cuddling or kissing another child), or imitates the actions of another child. The "response" is classified as "negative" when the child refuses to respond to a question or instruction from another child. Social behaviors coded as "positive" response include: a) positive emotions when the child expresses positive emotion by smiling or making eye contact with another child; b) positive touch when the child accepts the touch, follows another child and cuddles or caresses him/her, and c) imitation when the child replicates the movements or speech of another child. Responses coded as "negative" included a) avoidance-isolation, when the child looks away from another child or a common activity, moves away from his partner, pushes $\mathrm{him} / \mathrm{her}$, closes his eyes, and does not respond when his name is called; and b) aggressiveness, when the child pushes, pulls, or scares another child. The negative high-pitched verbalizations that are sometimes used by the child with autism and scare other children were also included in this particular category. ${ }^{10}$ In order to evaluate positive and negative social interaction, the method of continuous registration was used and each child was observed individually. Also, in order to increase the accuracy of observation, behaviors were defined explicitly, in addition to live observation, video observation was used to reduce the chance of missing the desired behaviors, and also evaluators were trained by a child psychologist who had the history of working with ASD children. Since each of the above factors, or a combination of them, can affect evaluations, reliability method was used for observers to show the reliability between live and video observation results. In order to determine reliability of frequency measurement of social interaction, the following formula was used.

\section{Reliability ratio $=$ the smallest recorded frequency $/$ the largest recorded frequency $\times 100$}

Reliability ratio between these two methods of social interaction evaluation was 0.92 .

\section{Gross motor skills}

Twelve gross motor skills including locomotor skills (running, galloping, hopping, leaping, jumping, and sliding) 
and object control skills (two-hand striking, stationary bouncing, catching, kicking, overhand throwing, and underhand rolling) were assessed using the qualitative test of Gross Motor Development-2 (TGMD-2). Each skill was measured twice and evaluated based on the presence (success; score 1) or absence (failure; score 0) of three to five qualitative performance criteria. The highest total raw score for both subjects is 48 with higher scores indicating improved performance. In this study, however, the raw TGMD-2 scores were used, as the normative TGMD-2 data collected for children in the United States may not be valid for children in other countries. ${ }^{37}$ The TGMD-2 offers great psychometric qualities to assess gross motor skill performance in children with normal development. Reliability and validity of the TGMD-2 in children with mild intellectual disabilities and learning disabilities have been confirmed by Ulrich. ${ }^{38}$

\section{Statistical analysis}

Mean and SD were calculated for each participant. A repeated measures ANCOVA was utilized to examine the effect of time (pre-test, post-test, and follow-up) and group (experimental groups and control) on each dependent variable while controlling for gender, Nonverbal intelligence Quotient (NIQ), Childhood Autism Rating Scale (CARS), body mass index (BMI), and age. A Bonferroni correction was applied to further evaluate the significant difference identified by the multiple comparisons analysis. We also checked typical assumptions of ANCOVA, such as normality and homogeneity of variance by Boxplot and Q-Q plot (residuals versus fitted values). The level of significance was set at 0.05 . All statistical analyses were performed by the R statistical software (version 3.4.3).

\section{Results}

\section{Demographic characteristics}

Table 1 lists information on the participants' characteristics. There was no significant difference between the groups in age $\mathrm{F}(3.42)=0.03, P=0.99$, NIQ F $(3,42)$ $=1.21, P=0.31$, as measured by Cattle Intelligence Scale for Children, BMI F $(3,42)=0.45, P=0.71$, and CARS F $(3,42)=1.21, P=0.31$. According to ASD prevalence estimates by gender, the majority of participants in both groups were male (45 males and 15 females). Table 2 provides means and SD of Gross Motor Skills scores, repetitive behaviors frequency, and positive and negative

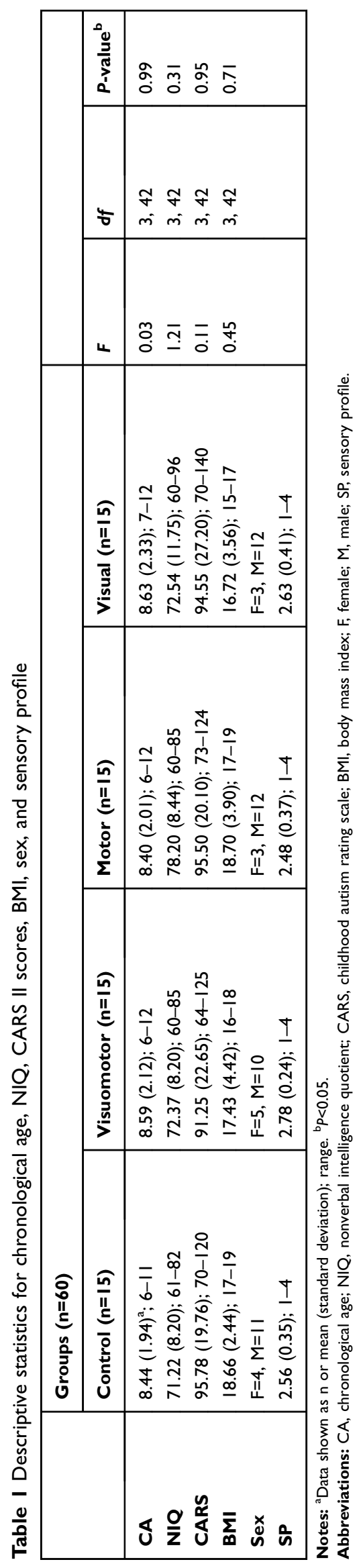




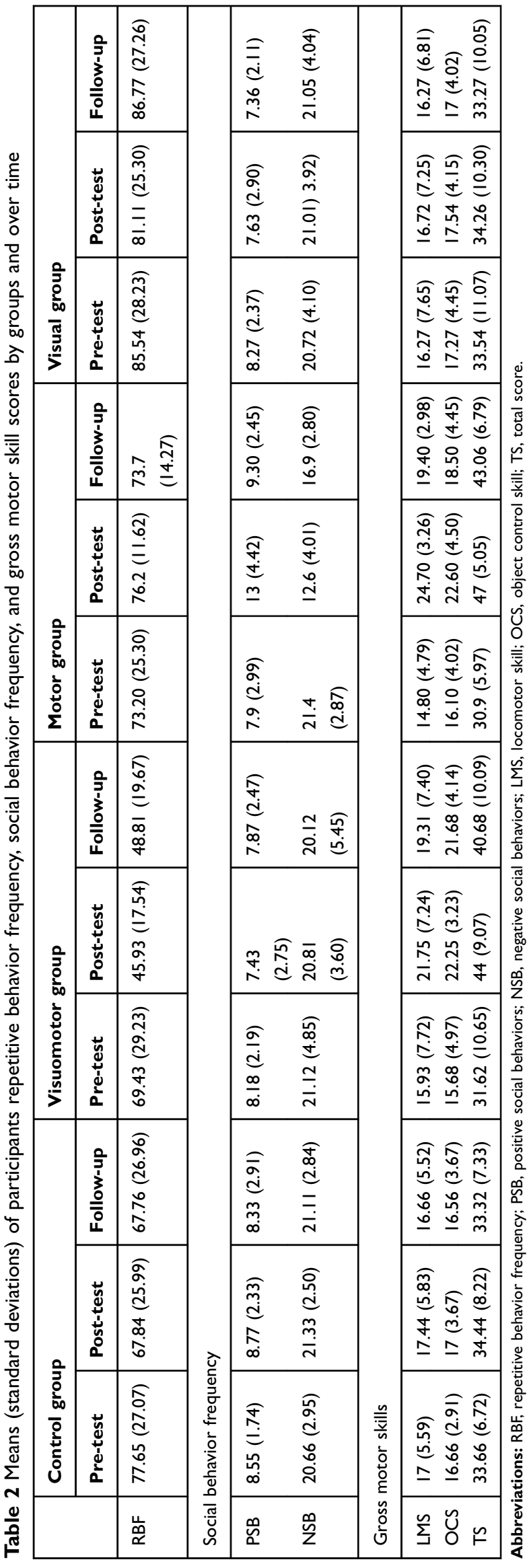

social behavior frequency for participants in all of the groups at the designated testing times.

\section{Effect of intervention on repetitive behaviors}

The repeated measures ANCOVA indicates that the model is significant at $0.05 \%$ level with control variables such as age, NIQ, CARS, sex, and BMI. The within-subject and between-subject effects were statistically significant at the level of 0.05. Moreover, the interaction of time and groups was significant $(P=0.02)$. The results are shown in Table 3 . To explore this interaction, Bonferroni comparison test was conducted.

As shown in Figure 1, there is no significant difference in the pre-test between groups with respect to repetitive behaviors frequency $(P>0.05)$. There was a statistical difference between visuomotor and other groups in post-test and follow-up $(P<0.05)$, where repetitive behaviors demonstrated a significant decline in this group. However, the repetitive behaviors took an upturn in the follow-up while visuomotor was not significantly different from the post-test. In the follow-up, nevertheless, repetitive behaviors exhibited a significant reduction in comparison with the pre-test or the other groups $(P>0.05)$.

\section{Effect of intervention on social behavior}

Repeated measure ANCOVA indicates that the model is significant at the level of $0.05 \%$ with control variables such as age, NIQ, CARS, sex, and BMI. The within-subject and between-subject effects were statistically significant at the level of $0.05 \%$. There was also a significant difference in time and group interaction on positive $(P=0.0002)$ and negative $(P=0.0003)$ social behavior. The results are shown in Table 4. We used Bonferroni comparison test to explore this interaction

Figure 2 does not show any significant differences in the pre-test between groups in their positive or negative social behaviors $(P>0.05)$. Significant differences in positive social behavior, however, were found between the motor group and other groups in post-test and follow-up $(P<0.05)$. Also, there was a statistically significant difference in the motor training group with respect to the negative behaviors $(P<0.05)$.

\section{Effect of intervention on gross motor skills}

Repeated measure ANCOVA indicates that the model is significant at the level of $0.05 \%$ with control variables 
Table 3 Repeated measure analysis variance of repetitive behavior frequency

\begin{tabular}{|l|l|l|l|l|l|l|}
\hline & Effect & df & Sum Sq & Mean Sq & F-value $^{\text {P-value }}$ \\
\hline RBF & Time & 2 & 2974 & 1487.1 & 6.9174 & 0.001 \\
& Group & 3 & 21,363 & 7121.1 & 33.1244 & 0.001 \\
& Timexgroup & 6 & 3259 & 543.1 & 2.5264 & 0.02 \\
\hline
\end{tabular}

Notes: ${ }^{\mathrm{a}} \mathrm{P}<0.05$.

Abbreviations: RBF, repetitive behavior frequency; df degrees of freedom; Sum Sq, sum of square; Mean Sq., mean of square.

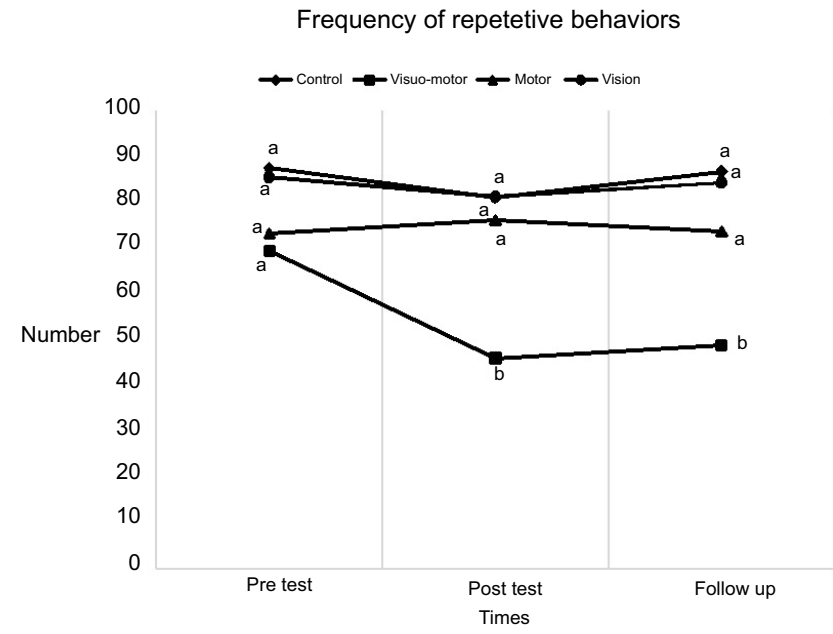

Figure I Group differences in pre-test, post-test, and follow-up of repetitive behaviors. Same letter in each row means that they are not significantly different.

such as age, NIQ, CARS, sex, and BMI. The within-subject and between-subject effects were statistically significant at the level of $0.05 \%$. Also, the interaction of time and groups was significant $(P<0.001)$, as shown by the results in Table 5. To explore this interaction, we performed Bonferroni comparison test

Figure 3 does not reveal any statistically significant difference between four groups regarding TGMD-2 total scores, locomotors skills, and object control skills in pre-test $(P>0.05)$. However, the post-test results suggest that both visual-motor and motor experimental groups outperformed the control and computer-based visual training groups in relation to the all gross motor variables. A comparison of groups in follow-up indicates that the visual-motor group had a significantly greater performance on total scores and object control skills compared to other groups $(P<0.05)$.

\section{Discussion}

The aim of this study was to assess which of visuomotor, group-based motor, or computer-based visual training had effect on the most core features of 6- to 12-year-old children with ASD which include gross motor skills, social communication, and repetitive behaviors. The results showed that visuomotor training had a significant effect on gross motor skills (post-test and follow-up) and frequency of repetitive behaviors (post-test and follow-up). Also, motor training had a significant effect on gross motor skills (post-test) and social communication (posttest). Our results also show that computer-based visual training had no significant effect on any core features of ASD and did not differ compared to control group. These results cannot be explained merely by general and developmental differences in age, intelligence, and autism severity as all of the above groups were identical with regard to these variables.

The results suggested a significant decline in repetitive behaviors of the visuomotor group in the post-test and follow-up. This finding in the visuomotor training group is consistent with those reported by Rose et al, ${ }^{26}$ who showed

Table 4 Repeated measure analysis variance of positive and negative social behaviors frequency

\begin{tabular}{|l|l|l|l|l|l|l|}
\hline & Effect & df & Sum Sq & Mean Sq & $\boldsymbol{F}_{\text {-value }}$ & $\boldsymbol{P}_{\text {-value }}$ \\
\hline PSB & Time & 2 & 18.36 & 9.18 & 11.71 & 0.0004 \\
& Group & 3 & 112.7 & 37.59 & 47.95 & 0.0002 \\
& Timexgroup & 6 & 130.72 & 21.78 & 27.79 & 0.0002 \\
\hline NSB & Time & 2 & 78.78 & 39.39 & 25.91 & 0.0004 \\
& Group & 3 & 355.3 & 118.34 & 77.84 & 0.0002 \\
& Timexgroup & 6 & 319.48 & 53.24 & 35.02 & 0.0003 \\
\hline
\end{tabular}

Notes: ${ }^{a} P<0.05$.

Abbreviations: PSB, positive social behaviors; NSB, negative social behaviors; DF, degrees of freedom; Sum Sq., sum of square; Mean Sq., mean of square. 


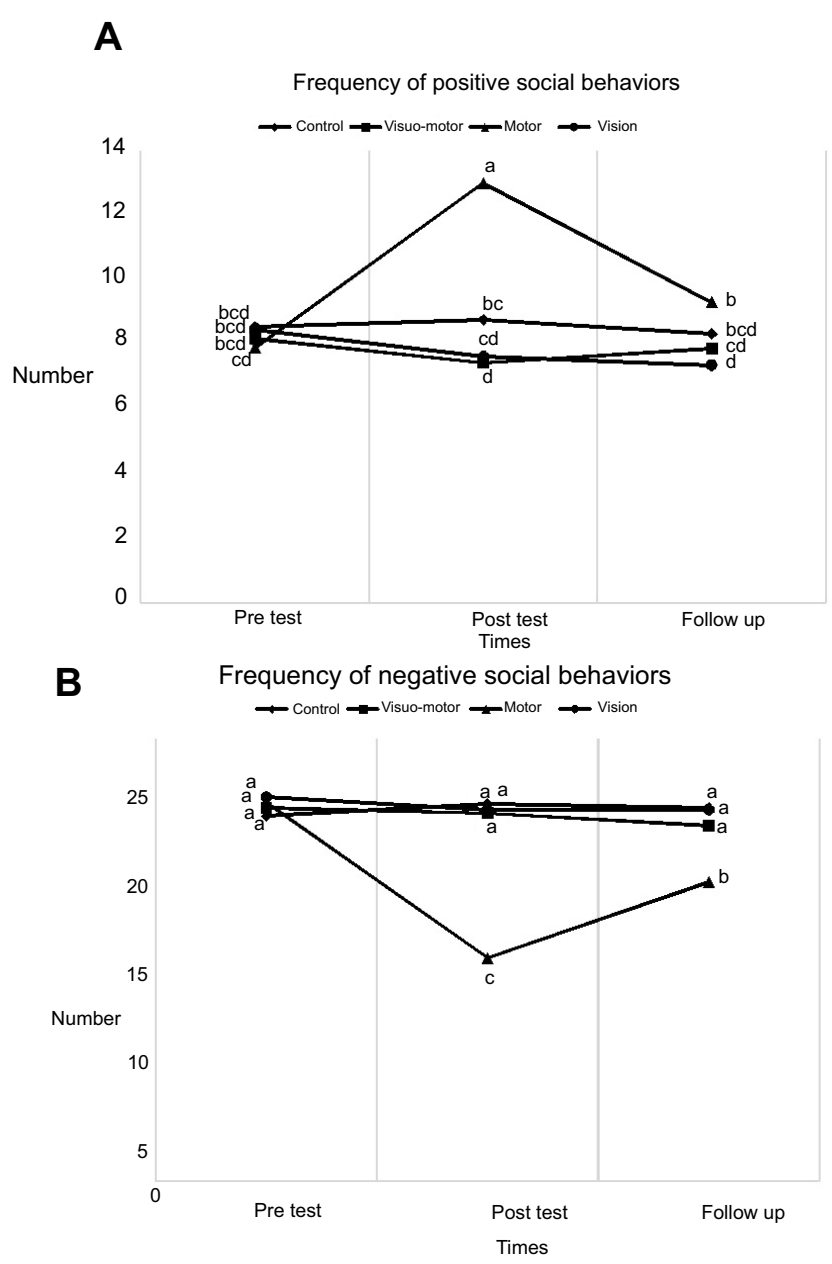

Figure 2 Group differences in pre-test, post-test, and follow-up of positive and negative social behavior frequency. Same letter in each row means that there are not significantly different.

that OPT can restrain repetitive behaviors in children with ASD, but they also reported the lasting effect of this kind of intervention is short, which is not consistent with our results. They noted that improvement in peripheral awareness and attention was responsible for constrained repetitive behavior. $^{26}$ On the other hand, our findings in the motor training group are in disagreement with the study of Baranek, ${ }^{24}$ who reported that motor training can mitigate repetitive behaviors by physiological change of the brain. In that review paper, motor training was considered as an aerobic training, whereas in our study, most of the motor trainings were motor perceptual training and the time of aerobic training was relatively short. By the mitigation of restricted and repetitive behaviors in the visuomotor of this study, it seems that visuomotor trainings can modify visual processing. The computer-based visual training group in our study only performed exercises that involved eye tracking and eye-hand coordination which did not have any significant effect on repetitive behavior frequency, but it seems that moving in the environment and spatial orientation are among the factors contributing to the improvement of body responses and visual processing of children with autism. Also, motor experience nurtures spatial-visual skills in children. ${ }^{39}$ Skeffington, the father of Behavioral Optometry, believes that the visual perception is a process that comes from the behavior of movement in the environment, experiences, and spatial orientation. ${ }^{40}$ In other words, the visual perception must be learned. Since vision processing in individuals with ASD poses a problem, they rely on deep body proprioceptive to gain more environmental information. Thus, they rock their bodies or flap their hands near the face. The improvement in visual processing seems to mitigate compensatory behaviors in these individuals, a hypothesis which is confirmed by the present study. Additionally, Ludlow et al $^{41}$ showed that low-level perceptual abnormalities may be responsible for autistic individuals' difficulties in attending and processing social and facial stimuli.

Table 5 Repeated measure analysis variance of gross motor skills

\begin{tabular}{|c|c|c|c|c|c|c|}
\hline & Effect & $d f$ & Sum Sq & Mean Sq & $F$-value & $P$-value ${ }^{a}$ \\
\hline \multirow[t]{3}{*}{ LMS } & Time & 2 & 386.3 & 193.13 & I58.84 & 0.001 \\
\hline & Group & 3 & 369 & $123.0 \mid$ & 101.17 & 0.001 \\
\hline & Timexgroup & 6 & 270 & 45 & 100.02 & 0.001 \\
\hline \multirow[t]{3}{*}{ OCS } & Time & 2 & 338.65 & 169.32 & 60.90 & 0.001 \\
\hline & Group & 3 & 300.72 & 100.23 & 36.05 & 0.001 \\
\hline & Timexgroup & 6 & 253.43 & 42.23 & 15.19 & 0.001 \\
\hline \multirow[t]{3}{*}{ TS } & Time & 2 & 1367.8 & 683.92 & II 3.342 & 0.001 \\
\hline & Group & 3 & 1448.7 & 482.89 & 80.02 & 0.001 \\
\hline & Timexgroup & 6 & 1043.2 & 137.86 & 28.81 & 0.001 \\
\hline
\end{tabular}

Notes: ${ }^{a} P<0.05$

Abbreviations: LMS, locomotor skill; OCS, object control skill; TS, total score; DF, degrees of freedom; Sum Sq., sum of square; Mean Sq., mean of square. 


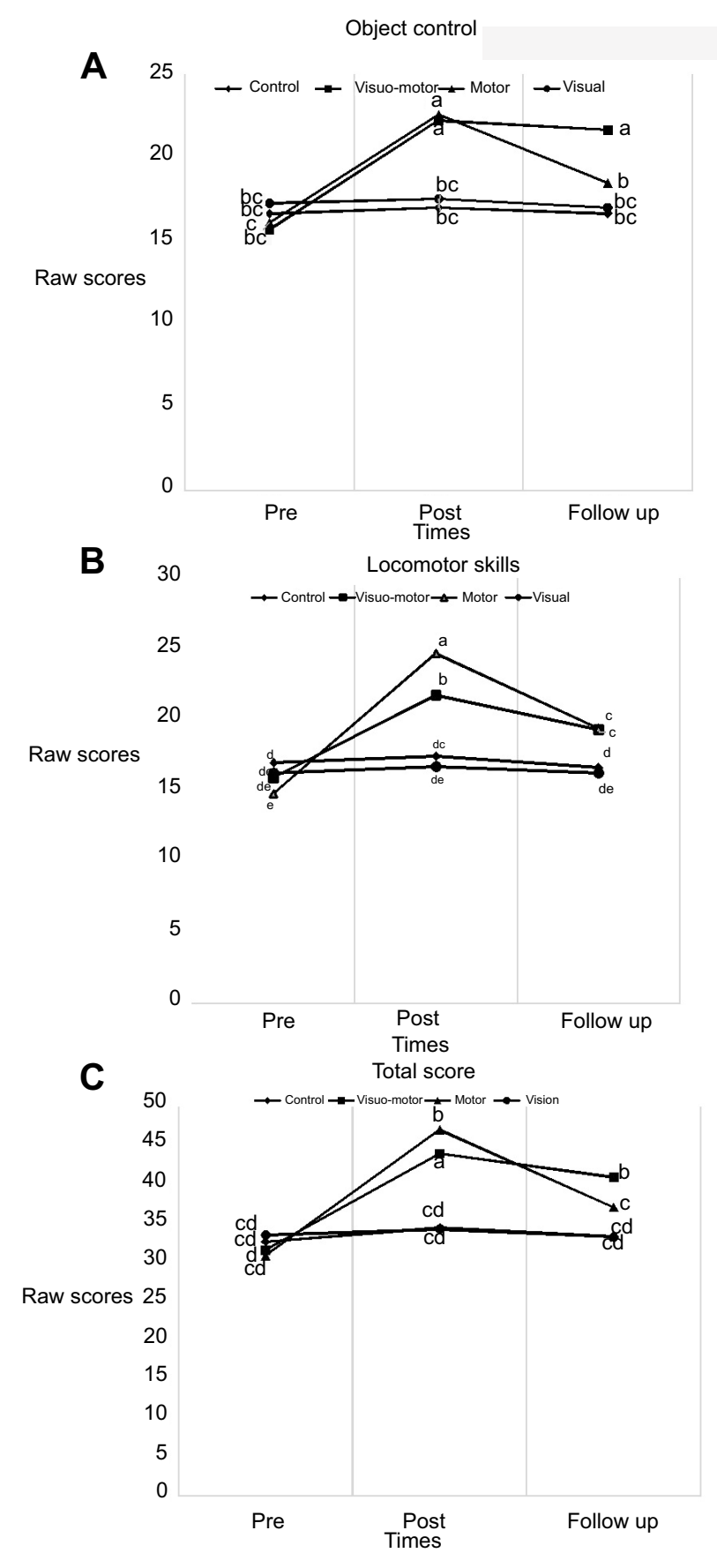

Figure 3 (A-C) Group differences in pre-test, post-test, and follow-up of gross motor skills. Same letter in each row means that there are not significantly different.

Social behaviors are complex and involve a number of causes that need to be investigated in order to successfully navigate the social environment. ${ }^{42}$ In this context, global information processing, due to dorsal stream pathway, is critically important. Given that individuals with ASD tend to have a narrow focus during information processing, they may miss important aspects of the social environment. Our results suggested that social behavior behaviors in the visuomotor group, which was aimed at modifying atypical visual processing, did not improve significantly in comparison with the control or computer-based visual training groups, which is in contrast with Hellendoorn's hypothesis, who maintained that atypical dorsal stream visual processing and social skills were related in young children with autism. Hence, many factors can contribute to social behavior. Hellendoorn used Viland social skill questionnaire for assessing social communication, which mostly assesses communications between a child and his/ her caregiver. ${ }^{11}$ In our study, however, we investigated social communication in the form of peer behaviors using an observational method under an ecological situation. Despite some improvements observed in repetitive and restricted behaviors and gross motor skill in our visuomotor group, which are probably due to the modification of visual processing, it seems that such improvements in visual processing have not significantly affected social communication impairment in children with autism. It is speculated that social communication is far from a simple dysfunction of the sensory processing. Nevertheless, our results suggested that social skills in motor training group improved significantly compared with the other groups. This provides further evidence that social communication skills are developed through direct experience of cooperative social skills with peers, and recognition of other people's feelings, ${ }^{10}$ which is consist with studies that are based on TOM. ${ }^{8-10}$ In our study, the motor group subjects received group-based training, in which they were engaged in peer-play organization, toys sharing, and assistance of one another. This kind of intervention probably has a positive effect on social responses. Our results in the motor training groups are consistent with the study according to which 60 -min sessions of physical activity twice a week for 8 consecutive weeks have a positive effect on social skills. ${ }^{43}$ Another difficulty that ASD children have to deal with is motor development delay, which may affect visual processing.

Children with ASD have been shown to develop motor development patterns later than their peers. ${ }^{44-47}$ However, we assumed that the visuomotor training would gain higher scores in gross motor skills in comparison with other groups following the intervention in a bid to satisfy the high demand for gross motor skills like catching ball, jumping, running, and appropriate visual processing. Although participants in visuomotor group and motor group witnessed significant improvement in gross motor development, we failed to obtain evidence for this hypothesis in the post-test results, although the follow- 
up results indicated otherwise. As can be observed in the follow-up, the visuomotor group was still significantly different in performance compared to follow-up results of the motor and other groups. The improved gross motor skills after visuomotor and motor training (post-test) were not surprising as researchers have shown that early motor skill instruction is effective and induces positive changes in the development of children's motor skills. ${ }^{24,48}$ The retention test after 2 months showed that visuomotor group was able to maintain the gross motor skill performance, which indicates the effectiveness of the intervention. It should be noted that the scores of motor skill intervention in post-test exhibited immediate motor learning (performance), with motor skills in the follow-up test showing more stable results in the development of motor skills (learning). ${ }^{46}$ According to the literature, motor skill problems of individuals with ASD are primarily related to the motor coordination, ${ }^{4-51}$ which is in agreement with our results since object control skills in TGMD-2 test are strongly dependent on motor coordination. Hence, it can be concluded from our results that visuomotor training as a motor-perceptual training had a positive learning effect on object control skills that need eye-hand/foot coordination. One explanation for this, as discussed in the literature, is that motor activities and enhancement sensory stimulate neural activities in a range of peripheral and central systems along with some subsequent molecular and cellular changes. ${ }^{52}$ Learning skills involve some structural changes in the nervous system, and since the basic activities in the visuomotor group contained visual processing stimulation, it seems that nervous system changes probably occurred in the visual cortex. ${ }^{53}$ This hypothesis, nonetheless, should be further investigated.

\section{Conclusion}

Our data provides preliminary evidences core features of ASD that support the effectiveness of visuomotor training on two of which with a positive maintenance effect for two month. Our results suggested improvements in repetitive behaviors and gross motor development, either in the post-test or the follow-up. However, it did not have any significant effect on social behavior. On the other hand, motor training group only demonstrated improvement in social behaviors (post-test) as well as significant improvement in post-test scores of gross motor skills. Given the improved social behavior of individuals in the motor training group due to group-based training protocols and also the positive effect of visuomotor training on gross motor skills and repetitive behaviors of children with $\mathrm{ASD}$, it seems that performing visuomotor training on a group-based training can provide an effective intervention, which exerts a significant effect on core features of children with ASD. The present study has several limitations that should be noted. Although we randomized subjects based on autism severity and cognition, we were not able to consider this distinction in our analysis due to our small sample size. Future studies can examine a large sample to evaluate the impact of potentially confounding variables on treatment outcomes. Furthermore, almost all of our participants demonstrated mild-to-moderate severity of autism. As such, future studies can apply this intervention to children with high severity of autism to determine whether the findings of this study can be generalized to that sample as well. Despite these limitations, this study provides evidence that support the effectiveness of this intervention as an adjunct to a comprehensive intervention program for children with ASD, who struggle with functional and behavioral challenges associated with visual information processing difficulties.

\section{Acknowledgments}

All expenses of the project were paid personally and no financial help was granted from any specific entity. This paper is prepared from a doctoral dissertation in motor development (Arabi M. The effect of visual-motor intervention on the social performance, stereotyped behaviors, gross motor skills and visual functions of children with autism spectrum disorder [Dissertation]. Mashhad: Ferdowsi University of Mashhad; 2019). Ultimately, we should thank the Welfare Organization of Razavi Khorasan Province and its autism institutes. We are grateful to the families who took part in our research. We also wish to thank teachers, coaches, and specialists who helped us in this study.

\section{Disclosure}

The authors report no conflicts of interest in this work.

\section{References}

1. Weinger PM, Zemon V, Soorya L, Gordon J. Low-contrast response deficits and increased neural noise in children with autism spectrum disorder. Neuropsychologia. 2014;63:10-18. doi:10.1016/j. neuropsychologia.2014.07.031

2. Feng H, Lo -Y-Y, Tsai S, Cartledge G. The effects of theory-of-mind and social skill training on the social competence of a sixth-grade student with autism. J Posit Behav Interv. 2008;10(4):228-242. doi: $10.1177 / 1098300708319906$

3. Levinson LJ, Reid G. The effects of exercise intensity on the stereotypic behaviors of individuals with autism. Adapt Phys Activ Q. 1993;10(3):255-268. doi:10.1123/apaq.10.3.255

4. Todd T. Teaching motor skills to individuals with autism spectrum disorders. J Phys Educ Recreat Dance. 2012;83(8):32-48. doi:10.10 80/07303084.2012.10598827 
5. Sowa M, Meulenbroek R. Effects of physical exercise on autism spectrum disorders: a meta-analysis. Res Autism Spectr Disord. 2012;6(1):46-57. doi:10.1016/j.rasd.2011.09.001

6. Greenaway R, Davis G, Plaisted-Grant K. Marked selective impairment in autism on an index of magnocellular function. Neuropsychologia. 2013;51(4):592-600. doi:10.1016/j.neuropsychologia.2013.01.005

7. Wolstencroft J, Robinson L, Srinivasan R, Kerry E, Mandy W, Skuse D. A systematic review of group social skills interventions, and metaanalysis of outcomes, for children with high functioning ASD. $J$ Autism Dev Disord. 2018;48(7):2293-2307. doi:10.1007/s10803018-3485-1

8. Schneider N, Goldstein H, Parker R. Social skills interventions for children with autism: a meta-analytic application of percentage of all non-overlapping data (PAND). Evid Based Commun Assess Interv. 2008;2(3):152-162. doi:10.1080/17489530802505396

9. Pan C-Y. Effects of water exercise swimming program on aquatic skills and social behaviors in children with autism spectrum disorders. Autism. 2010;14(1):9-28. doi:10.1177/1362361309339496

10. Oke NJ, Schreibman L. Training social initiations to a high-functioning autistic child: assessment of collateral behavior change and generalization in a case study. J Autism Dev Disord. 1990;20(4):479497.

11. Hellendoorn A, Langstraat I, Wijnroks L, Buitelaar JK, van Daalen E, Leseman PP. The relationship between atypical visual processing and social skills in young children with autism. Res Dev Disabil. 2014;35 (2):423-428. doi:10.1016/j.ridd.2013.11.012

12. Grinter EJ, Maybery MT, Badcock DR. Vision in developmental disorders: is there a dorsal stream deficit? Brain Res Bull. 2010;82 (3-4):147-160. doi:10.1016/j.brainresbull.2010.02.016

13. Bakroon A, Lakshminarayanan V. Visual function in autism spectrum disorders: a critical review. Clin Exp Optom. 2016;99(4):297-308. doi:10.1111/cxo.12383

14. Freeth M, Foulsham T, Kingstone A. What affects social attention? Social presence, eye contact and autistic traits. PLoS One. 2013;8(1): e53286. doi:10.1371/journal.pone.0053286

15. Nation K, Penny S. Sensitivity to eye gaze in autism: is it normal? Is it automatic? Is it social? Dev Psychopathol. 2008;20(1):79-97. doi: $10.1017 / \mathrm{S} 0954579408000047$

16. Casartelli L, Chiamulera C. The motor way: clinical implications of understanding and shaping actions with the motor system in autism and drug addiction. Psychobiology. 2016;16(2):191-206.

17. Hashemi M, Roonasi A, Saboonchi R, Salehian MH. Effect of selected physical activities on social skills among 3-6 years old children. Life Sci J. 2012;9(4):4267-4271.

18. Park M. Pleasure, throwing breaches, and embodied metaphors: tracing transformations-in-participation for a child with autism to a sensory integration-based therapy session. OTJR. 2012;32 (1_suppl):S34-S47. doi:10.3928/15394492-20110906-05

19. Bernard KM. The Play and Learn (PAL) Program for Enhancing Social Participation and Peer Interaction for Children with Special Needs: A Program Development Plan. [Dissertation]. Toledo: University of Toledo; 2013.

20. Miltenberger CA, Charlop MH. Increasing the athletic group play of children with autism. J Autism Dev Disord. 2014;44(1):41-54. doi:10.1007/s10803-013-1850-7

21. Liu T, Breslin CM. Fine and gross motor performance of the MABC-2 by children with autism spectrum disorder and typically developing children. Res Autism Spectr Disord. 2013;7(10):12441249. doi:10.1016/j.rasd.2013.07.002

22. Gowen E, Hamilton A. Motor abilities in autism: a review using a computational context. J Autism Dev Disord. 2013;43(2):323-344. doi:10.1007/s10803-012-1574-0

23. Lang R, Koegel LK, Ashbaugh K, Regester A, Ence W, Smith W. Physical exercise and individuals with autism spectrum disorders: a systematic review. Res Autism Spectr Disord. 2010;4(4):565-576. doi:10.1016/j.rasd.2010.01.006
24. Baranek GT. Efficacy of sensory and motor interventions for children with autism. J Autism Dev Disord. 2002;32(5):397-422.

25. Petrus C, Adamson SR, Block L, Einarson SJ, Sharifnejad M, Harris SR. Effects of exercise interventions on stereotypic behaviours in children with autism spectrum disorder. Physiother Can. 2008;60 (2):134-145. doi:10.3138/physio.60.2.134

26. Rose M, Torgerson NG. A behavioral approach to vision and autism. JOVD. 1994;25:269-275.

27. Mottron L, Mineau S, Martel G, et al. Lateral glances toward moving stimuli among young children with autism: Early regulation of locally oriented perception? Dev Psychopathol. 2007;19(01):23-36. doi:10.1017/S0954579407070022

28. Coulter RA. Understanding the visual symptoms of individuals with autism spectrum disorder (ASD). OVD. 2009;40(3):164.

29. Jiang YV, Swallow KM. Body and head tilt reveals multiple frames of reference for spatial attention. J Vis. 2013;13(13):9. doi:10.1167/ 13.13 .9

30. Kern L, Koegel RL, Dyer K, Blew PA, Fenton LR. The effects of physical exercise on self-stimulation and appropriate responding in autistic children. J Autism Dev Disord. 1982;12(4):399-419.

31. Hurst CM, Van de Weyer S, Smith C, Adler PM. Improvements in performance following optometric vision therapy in a child with dyspraxia. Ophthalmic Physiol Opt. 2006;26(2):199-210. doi:10.11 11/j.1475-1313.2006.00366.x

32. Zoia S, Castiello U, Blason L, Scabar A. Reaching in children with and without developmental coordination disorder under normal and perturbed vision. Dev Neuropsychol. 2005;27(2):257-273. doi:10.12 07/s15326942dn2702_4

33. Coetzee D, Pienaar AE. The effect of visual therapy on 7-to 8-year old children with Developmental Coordination Disorder (DCD). AJPHES. 2017;2017(Supplement 1.1):17-31.

34. Cheatum BA, Hammond AA. Physical activities for improving children's learning and behavior: a guide to sensory motor development. 2end ed Human Kinetics. 2000:45-72.

35. Nigg C, Geller K, Adams P, Hamada M, Hwang P, Chung R. Successful dissemination of fun 5 - a physical activity and nutrition program for children. Transl Behav Med. 2012;2(3):276-285. doi:10.1007/s13142-012-0120-0

36. Strain PS, Shores RE. Social interaction development among behaviorally handicapped preschool children: research and educational implications. Psychol Sch. 1977;14(4):493-502. doi:10.1002/15206807(197710)14:4<493::AID-PITS2310140422>3.0.CO;2-W

37. Green D, Charman T, Pickles A, et al. Impairment in movement skills of children with autistic spectrum disorders. Dev Med Child Neurol. 2009;51(4):311-316. doi:10.1111/j.1469-8749.2008.03242.x

38. Ulrich DA. The test of gross motor development-3 (TGMD-3): administration, scoring, and international norms. Spor Bilimleri Dergisi. 2013;24(2):27-33.

39. Thelen E. Motor development as foundation and future of developmental psychology. Int J Behav Dev. 2000;24(4):385-397. doi:10.1080/016502500750037937

40. Kaplan M. Seeing through New Eyes: Changing the Lives of Children with Autism, Asperger Syndrome and Other Developmental Disabilities through Vision Therapy. London: essica Kingsley Publishers; 2005.

41. Ludlow AK, Taylor-Whiffen E, Wilkins AJ. Coloured filters enhance the visual perception of social cues in children with autism spectrum disorders. ISRN Neurol. 2012;(4).

42. Hill TL, Varela RE, Kamps JL, Niditch LA. Local processing and social skills in children with autism spectrum disorders: the role of anxiety and cognitive functioning. Res Autism Spectr Disord. 2014;8 (9):1243-1251. doi:10.1016/j.rasd.2014.06.005

43. Duronjić M, Válková $H$. The influence of early intervention movement programs on motor skills development in preschoolers with autism spectrum disorder (case studies). Acta Gymnica. 2010;40(2):37-45. 
44. Berkeley SL, Zittel LL, Pitney LV, Nichols SE. Locomotor and object control skills of children diagnosed with autism. Adapt Phys Activ $Q$. 2001;18(4):405-416. doi:10.1123/apaq.18.4.405

45. Pan C-Y, Tsai C-L, Chu C-H. Fundamental movement skills in children diagnosed with autism spectrum disorders and attention deficit hyperactivity disorder. J Autism Dev Disord. 2009;39 (12):1694. doi:10.1007/s10803-009-0813-5

46. Vernadakis N, Papastergiou M, Zetou E, Antoniou P. The impact of an exergame-based intervention on children's fundamental motor skills. Comput Educ. 2015;83:90-102. doi:10.1016/j. compedu.2015.01.001

47. Mache MA, Todd TA. Gross motor skills are related to postural stability and age in children with autism spectrum disorder. Res Autism Spectr Disord. 2016;23:179-187. doi:10.1016/j.rasd.2016.01.001

48. Huettig C. Physical Activities for Improving Children's Learning and Behavior: A Guide to Sensory Motor Development. Champaign: Human Kinetics Pub; 2000.
49. Fazlioglu Y, Gunsen MO. Sensory motor development in autism. Mohammad-Reza Mohammadi MR, editor. In: A Comprehensive Book on Autism Spectrum Disorders. London: InTech; 2011.

50. Staples KL, Reid G. Fundamental movement skills and autism spectrum disorders. J Autism Dev Disord. 2010;40(2):209-217. doi:10.1007/s10803-009-0854-9

51. Logan S, Robinson L, Wilson A, Lucas W. Getting the fundamentals of movement: a meta-analysis of the effectiveness of motor skill interventions in children. Child Care Health Dev. 2012;38(3):305315. doi:10.1111/j.1365-2214.2011.01307.x

52. Hannan A. Environmental enrichment and brain repair: harnessing the therapeutic effects of cognitive stimulation and physical activity to enhance experience-dependent plasticity. Neuropathol Appl Neurobiol. 2014;40(1):13-25. doi:10.1111/nan.12102

53. Pourtois G, Rauss KS, Vuilleumier P, Schwartz S. Effects of perceptual learning on primary visual cortex activity in humans. Vision Res. 2008;48(1):55-62. doi:10.1016/j.visres.2007.10.027 


\section{Supplementary material}

Table SI Exercises of visuomotor training protocol

\begin{tabular}{|c|c|c|c|}
\hline Sessions I-9 & Sessions 9-16 & Sessions 16-23 & Sessions $23-30$ \\
\hline Body awareness & Spatial awareness & Peripheral vision & Pursuits and saccades \\
\hline Angles in snow & Walking on the tube & $\begin{array}{l}\text { Walking on the certain } \\
\text { road }\end{array}$ & Marble in pan \\
\hline Pointer dog & Jump on the target & Hitting the hanging ball & Marsden ball \\
\hline Swimming in place & Making road & $\begin{array}{l}\text { Playing with different size } \\
\text { of ball }\end{array}$ & Marble roll \\
\hline Walking rail & $\begin{array}{l}\text { Making spider web with } \\
\text { cotton }\end{array}$ & Playing with balloon & Rotating pegboard \\
\hline Hopscotch & Crawl on the circle & Catching the moving light & Penlights saccades \\
\hline Chalkboard circles & Catching magic scarf & Moving ball with spoon & Sticker saccades \\
\hline Windshield wipers & Ball rolling & $\begin{array}{l}\text { Drawing on the } \\
\text { blackboard }\end{array}$ & Groffman tracking \\
\hline Jumping jacks & Swedish swimming & & Hart chart \\
\hline Soldier crawl & & & Pegboard \\
\hline
\end{tabular}

Note: Adapted with permission from Hurst CM, Van de Weyer S, Smith C, Adler PM. Improvements in performance following optometric vision therapy in a child with dyspraxia. Ophthalmic Physiol Opt. 2006;26(2):199-210. @ 2006 The College of Optometrists. ${ }^{31}$ And from Coetzee D, Pienaar AE. The effect of visual therapy on 7-to 8-year old children with Developmental Coordination Disorder (DCD). AJPHES. 2017;2017(Supplement 1.1):17-31. @ 2013 Elsevier Ltd. All rights reserved. ${ }^{33}$

\section{Publish your work in this journal}

Neuropsychiatric Disease and Treatment is an international, peerreviewed journal of clinical therapeutics and pharmacology focusing on concise rapid reporting of clinical or pre-clinical studies on a range of neuropsychiatric and neurological disorders. This journal is indexed on PubMed Central, the 'PsycINFO' database and CAS, and is the official journal of The International Neuropsychiatric Association (INA). The manuscript management system is completely online and includes a very quick and fair peer-review system, which is all easy to use. Visit http://www.dovepress.com/testimonials.php to read real quotes from published authors. 